\title{
Combination Therapy of Capecitabine with Cyclophosphamide as a Second-Line Treatment after Failure of Paclitaxel plus Bevacizumab Treatment in a Human Triple Negative Breast Cancer Xenograft Model
}

\author{
Mieko Yanagisawa, Keigo Yorozu, Mitsue Kurasawa, Yoichiro Moriya*, Naoki Harada \\ Product Research Department, Chugai Pharmaceutical Co., Ltd., Kamakura, Japan. \\ Email: ${ }^{*}$ moriyayui@chugai-pharm.co.jp \\ Received July $25^{\text {th }}, 2013$; revised August $21^{\text {st }}, 2013$; accepted August $26^{\text {th }}, 2013$ \\ Copyright (C) 2013 Mieko Yanagisawa et al. This is an open access article distributed under the Creative Commons Attribution Li- \\ cense, which permits unrestricted use, distribution, and reproduction in any medium, provided the original work is properly cited.
}

\begin{abstract}
We examined the antitumor efficacy of the capecitabine (CAPE) plus cyclophosphamide (CPA) combination as a $2^{\text {nd }}$-line therapy after paclitaxel (PTX) plus bevacizumab (BEV) treatment in a xenograft model of human triple negative breast cancer (TNBC) cell line, MX-1. After tumor growth was confirmed, PTX (20 mg/kg; i.v.) + BEV (5 mg/kg; i.p.) treatment was started (Day 1). Each agent was administered once a week for 5 weeks and tumor regression was observed for at least the first 3 weeks. For $2^{\text {nd }}$-line treatment, we selected mice in which the tumor volume had increased from day 29 to day 36 and was within $130-250 \mathrm{~mm}^{3}$ on day 36. After randomization of mice selected on day 36 , CPA $(10 \mathrm{mg} / \mathrm{kg}$; p.o.) and CAPE (539 mg/kg; p.o.) were administered daily for 14 days (days 36 - 49), followed by cessation of the drugs for 1 week. The tumor growth on day 57 was significantly suppressed in the CPA, CAPE and CAPE + CPA groups as compared with the control group $(\mathrm{p}<0.05)$. Furthermore, the antitumor activity on day 57 of CAPE + CPA was significantly stronger than that of CPA or CAPE alone $(\mathrm{p}<0.05)$. The thymidine phosphorylase $(\mathrm{TP})$ level in tumor tissue was evaluated by immunohistochemistry on day 50, and was significantly higher in the CPA group than those in the control group $(\mathrm{p}<0.05)$. Upregulation of TP in tumor tissues by CPA treatment would increase the 5-FU level in tumor tissues treated with CAPE. This would explain the possible mechanism that made CAPE + CPA superior to CAPE alone in the $2^{\text {nd }}$-line treatment. Our preclinical results suggest that the CAPE + CPA combination therapy may be effective as $2^{\text {nd }}$-line therapy after disease progression in PTX + BEV $1^{\text {st }}$-line treatment for TNBC patients.
\end{abstract}

Keywords: Triple Negative Breast Cancer; Capecitabine; Cyclophosphamide; Bevacizumab; Paclitaxel; Xenograft Model

\section{Introduction}

Bevacizumab (BEV) is a genetically engineered humanized monoclonal antibody derived from murine anti-human vascular endothelial growth factor (VEGF) monoclonal antibody A4.6.1 [1,2]. It binds specifically to human VEGF, thereby blocking the binding of VEGF to VEGF receptors expressed on vascular endothelial cells. By blocking the biological activity of VEGF [3], antihuman VEGF antibodies such as BEV inhibit neovascularization in tumor tissues and thus suppress tumor growth [1,4-9]. Paclitaxel (PTX) binds to $\beta$-tubulin and stabilizes microtubules, which represses the dynamic instability of spindle microtubules and results in blocking

"Corresponding author. the cell cycle at the metaphase-to-anaphase transition [10].

In clinical, BEV in combination with PTX (PTX + $\mathrm{BEV})$ significantly prolonged progression-free survival as compared with paclitaxel alone in the $1^{\text {st }}$-line treatment of metastatic breast cancers [11]. However, which treatment modality is effective as a $2^{\text {nd }}-$ line therapy after progressive disease of PTX + BEV treatment is controversial. On the other hand, combination therapy of capecitabine (N4-pentyloxycarbonyl-5'-deoxy-5-fluorocytidine, CAPE) plus cyclophosphamide (CPA) is considered to be effective for patients with HER2-negative metastatic breast cancer who have been treated with anthracyclines [12]. CAPE is an oral fluoropyrimidine drug widely used for breast cancers which generates the active substance 5-FU in tumors by a three-step cascade of enzymes lo- 
cated in the liver and tumors. The final step is the conversion of 5'-DFUR, an intermediate metabolite, to 5-FU by thymidine phosphorylase (TP), which is highly expressed in tumors. Therefore, in CAPE treatment, tumor tissues that have higher expression levels of TP would be expected to have higher levels of 5-FU. Indeed, it has been reported that the antitumor activity of CAPE did correlate with TP levels in tumor in xenograft models, whereas that of 5-FU did not $[13,14]$. Some antitumor modalities, such as CPA, taxanes, oxaliplatin, erlotinib, and radiation, have been reported to increase the levels of TP in tumors in xenograft models and to show significantly more potent antitumor activity in combination with CAPE than each agent or treatment as a monotherapy [15-20].

In this study, we examined the antitumor efficacy of the CAPE + CPA combination as a $2^{\text {nd }}-$ line therapy after disease progression in PTX + BEV $1^{\text {st }}$-line treatment in a xenograft model. For this purpose, we used a MX-1 human triple negative breast cancer (TNBC) cell xenograft model because, as we have previously reported, treatment with the PTX + BEV combination in this model showed higher antitumor activity than PTX or BEV alone [8] and, thanks to CPA upregulation of TP, CAPE + CPA showed a significant antitumor activity as a $1^{\text {st }}$ line treatment [15].

\section{Materials and Methods}

\subsection{Antitumor Drugs and Reagents}

BEV and CAPE were obtained from F. Hoffmann-La Roche, Ltd. (Basle, Switzerland). Human IgG (HuIgG) was purchased from MP Biomedicals, LLC (Solon, OH, USA). BEV and HuIgG were diluted with saline and were administered intraperitoneally (i.p.). PTX was commercially obtained from Wako Pure Chemical Industries, Ltd. (Osaka, Japan). PTX was dissolved in Cremophor EL-ethanol solution (1:1) and diluted 1:10 with saline just before intravenous (i.v.) administration. Cremophor EL-ethanol solution (1:1) diluted 1:10 with saline was administered as the PTX vehicle. Cremophor EL was purchased from Sigma-Aldrich Corp. (St. Louis, MO, USA). CPA, which was purchased from Shionogi \& Co., Ltd. (Osaka, Japan), was diluted with distilled water (DW) and administered orally (p.o.). DW was administered as the CPA vehicle. CAPE was suspended in 40 mmoles $/ \mathrm{L}$ citrate buffer ( $\mathrm{pH}$ 6.0) containing $5 \%$ gum arabic as the vehicle and given p.o. The $40 \mathrm{mmoles} / \mathrm{L}$ citrate buffer $(\mathrm{pH} 6.0)$ containing $5 \%$ gum arabic was administered as the CAPE vehicle.

\subsection{Animals}

Five-week-old female BALB-nu/nu (CAnN.Cg-Foxn1 < $\mathrm{nu}>/ \mathrm{CrlCrlj} \mathrm{nu/nu)} \mathrm{mice} \mathrm{were} \mathrm{obtained} \mathrm{from} \mathrm{Charles}$ River Laboratories Japan, Inc. (Kanagawa, Japan). All mice were housed in a pathogen-free environment under controlled conditions (temperature $20^{\circ} \mathrm{C}-26^{\circ} \mathrm{C}$, humidity $30 \%-70 \%$, light/dark cycle 12 hours $/ 12$ hours). Chlorinated water and irradiated food (CE-2; Clea Japan, Inc., Tokyo, Japan) were provided ad libitum. All mice were allowed to acclimatize and recover from shipping-related stress for 11 days prior to the study. The health of the mice was monitored by daily observation. The protocol was reviewed by the Institutional Animal Care and Use Committee of Chugai Pharmaceutical Co., Ltd. and all mouse experiments were performed in accordance with the Guidelines for the Accommodation and Care of Laboratory Animals promulgated in Chugai Pharmaceutical Co., Ltd.

\subsection{MX-1 Human Breast Cancer Xenograft Model}

The MX-1 human breast cancer cell line was kindly provided by Dr T. Tashiro (Cancer Chemotherapy Center, Japanese Foundation for Cancer Research, Tokyo, Japan). A piece of minced MX-1 tumor (approx. $10 \mathrm{~mm}^{3}$ ) was inoculated subcutaneously into the right flank region of each mouse.

\section{4. $1^{\text {st }}$-Line Treatment in the MX-1 Model}

Nineteen days after the MX-1 inoculation, mice bearing a tumor of $200-800 \mathrm{~mm}^{3}$ in volume were selected and were randomly allocated (day 1) to control (5 mice) or PTX + BEV treatment group (158 mice). As a $1^{\text {st }}$-line treatment, PTX $(20 \mathrm{mg} / \mathrm{kg}$, i.v. $)$ with BEV (5 mg/kg, i.p. $)$ was administered weekly for 5 weeks starting from day 1 . HuIgG $(5 \mathrm{mg} / \mathrm{kg})$ and PTX vehicle were administered in the control group.

\section{5. $2^{\text {nd }}$-Line Treatment and the Evaluation of Antitumor Efficacy}

For $2^{\text {nd }}$-line treatment, mice bearing a tumor that was 130 $-250 \mathrm{~mm}^{3}$ on day 36 and that had increased between day 29 and day 36 were selected. The selected mice were randomized on day 36 into 4 groups (control $\left[2^{\text {nd }} \mathrm{L}\right]$, CAPE, CPA, and CAPE + CPA; 6 mice per group) for the evaluation of antitumor efficacy, and 2 groups (control $\left[2^{\text {nd }} \mathrm{L}\right], \mathrm{CPA} ; 6$ mice per group) for TP analysis. CPA at $10 \mathrm{mg} / \mathrm{kg}$ and CAPE at $539 \mathrm{mg} / \mathrm{kg}$ (MTD) $[13,14]$ were given p.o. daily for 14 days (day 36 to 49), followed by cessation of the drugs for 1 week. The antitumor efficacy was evaluated by tumor volume (TV) and the percentage of tumor growth inhibition (TGI\%) on day 57. The TV was estimated using the equation $\mathrm{V}=$ $\mathrm{ab}^{2} / 2$, where $\mathrm{a}$ and $\mathrm{b}$ are the length and width of the tu- 
mor, respectively. TGI\% was calculated as follows: TGI $\%=[1-$ (mean change in TV in each group treated with antitumor drugs/mean change in TV in control group)] $\times 100$. TV and body weight were monitored twice a week starting from the first day of the treatment.

\subsection{Immunohistochemistry (IHC) of $T P$ in Tumor Tissues from $2^{\text {nd }}$-Line Treatment}

After mice from the $1^{\text {st }}$-line treatment had been randomly allocated to CPA and control groups (6 mice per group). CPA or CPA vehicle (DW) was given daily for 14 days (day 36 to 49 ) as the $2^{\text {nd }}-$ line treatment. The tumors were excised on day 50 , and $4 \mu \mathrm{m}$-thick sections were prepared from paraffin-embedded formalin-fixed tissues. IHC of TP was performed using anti-TP antibody (AntiTYMP, rabbit monoclonal antibody; SIGMA Life Science, MO, USA) and peroxidase-labeled polymer-horseradish peroxidase (HRP) conjugated goat anti-rabbit immunoglobulins (Envision + System-HRP-DAB; Dako, Tokyo).

IHC was evaluated by scoring the positive staining area and positive staining strength in each mouse in CPA post-PTX+BEV or control group post-PTX+BEV. Scores are as follows: -, negative; \pm , very slightly positive; +, slightly positive; ++, moderately positive; +++, markedly positive. In order to perform a statistical analysis, the IHC scores,,,- \pm+++ and +++ were quantified as $0,1,2$, 3 and 4 , respectively.

\subsection{Statistical Analysis}

Statistical analysis of TV and IHC scores was performed using the Wilcoxon test (SAS preclinical package, SAS Institute, Inc., Tokyo, Japan). Differences were considered to be significant at $\mathrm{p}<0.05$.

\section{Results}

\subsection{Antitumor Activity of $1^{\text {st }}$-Line and $2^{\text {nd }}$-Line Treatment}

During $1^{\text {st }}$-line treatment, an obvious antitumor effect was observed in the PTX + BEV group, as was seen in the previous study [8]. As for the $2^{\text {nd }}-$ line treatment, the average TV in each group on the starting day of $2^{\text {nd }}$-line treatment (day 36) was $182-184 \mathrm{~mm}^{3}$. On day 57, the TV (mean \pm SD) of each group was as follows: control $\left[2^{\text {nd }} \mathrm{L}\right]$ group, $2721 \pm 772 \mathrm{~mm}^{3}$; CAPE group, $1325 \pm 294$ $\mathrm{mm}^{3}$; CPA group, $1665 \pm 314 \mathrm{~mm}^{3}$; and CAPE $+\mathrm{CPA}$ group, $214 \pm 42 \mathrm{~mm}^{3}$. TGI\% on day 57 was $55 \%$ in CAPE group, $42 \%$ in CPA group, and $99 \%$ in CAPE + CPA group. The TV of the CPA, CAPE, and CAPE + CPA groups was significantly lower compared to that of the control $\left[2^{\text {nd }} \mathrm{L}\right]$ group $(\mathrm{p}<0.05$, Figure 1$)$. It is note- worthy that the antitumor activity of the CAPE + CPA group was significantly higher than that of the CPA or CAPE groups $(p<0.05$, Figure 1).

\subsection{IHC of TP in Tumor Tissue}

The results of IHC on TP in tumor tissues obtained on day 50 are shown in Figure 2 and Table 1. The score of positive staining area in the CPA group was significantly higher than that of the control $\left[2^{\text {nd }} \mathrm{L}\right]$ group $(\mathrm{p}<0.05)$. The score of positive staining strength of the CPA group was also significantly higher than that of the control $\left[2^{\text {nd }} L\right]$ group $(\mathrm{p}<0.05)$.

\section{Discussion}

In a phase III trial (E2100), BEV in combination with PTX significantly prolonged progression-free survival and increased the objective response rate compared with PTX alone in patients with metastatic breast cancer [11]. On the other hand, combination therapy of CAPE + CPA is considered to be effective for HER2-negative metastatic breast cancer [12]. In preclinical study, using an MX-1 xenograft model, it has been reported that antitu-

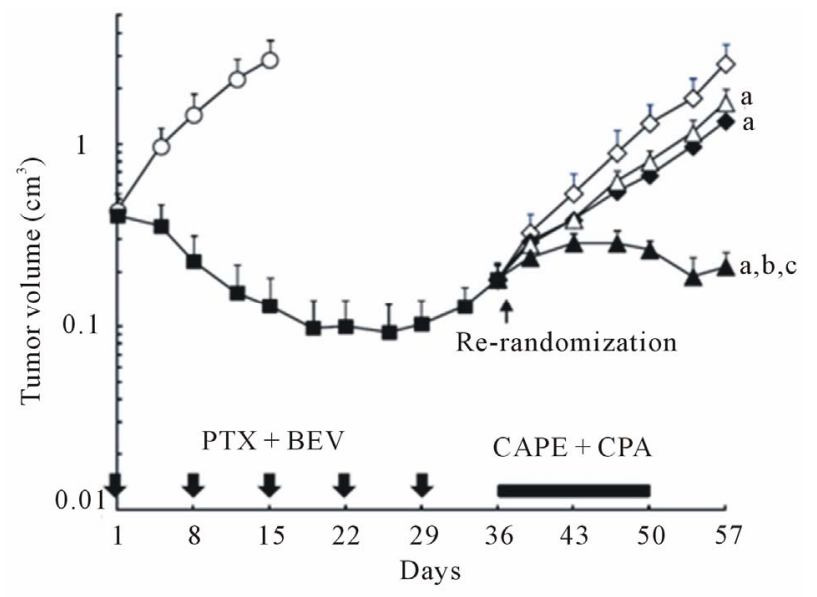

Figure 1. Antitumor activity of CAPE in combination with CPA as $2^{\text {nd }}$-line treatment, after $1^{\text {st }}$-line treatment with PTX and BEV. PTX at $20 \mathrm{mg} / \mathrm{kg}$ (i.v.) and BEV at $5 \mathrm{mg} / \mathrm{kg}$ (i.p.) were administered weekly for 5 weeks starting from day 1 . For $2^{\text {nd }}$-line treatment, mice bearing a tumor of 130 - 250 $\mathrm{mm}^{3}$ in volume on day 36 that had increased between day 29 and day 36 were selected. The mice were randomized into 4 groups of 6 mice each on day 36 as follows; control $\left[2{ }^{\text {nd }} \mathrm{L}\right], \mathrm{CPA}, \mathrm{CAPE}, \mathrm{CAPE}+\mathrm{CPA}$. CPA at $10 \mathrm{mg} / \mathrm{kg}$ and CAPE at $539 \mathrm{mg} / \mathrm{kg}$ were orally administered daily for 14 days followed by cessation of the drugs for 1 week. control (open circles), PTX + BEV (blocked squares), control [ $\left.2^{\text {nd }} \mathrm{L}\right]$ (open diamonds), CPA (open triangles), CAPE (blocked diamonds), CAPE + CPA (blocked triangles). Data points represent TV average + SD. (a) $p<0.05$ vs control $\left[2^{\text {nd }} L\right]$ group; (b) $p<0.05$ vs CPA group; (c) $p<0.05$ vs CAPE group by Wilcoxon test. 
Combination Therapy of Capecitabine with Cyclophosphamide as a Second-Line Treatment after Failure of Paclitaxel plus Bevacizumab Treatment in a Human Triple Negative Breast Cancer Xenograft Model

Table 1. IHC score of TP.

\begin{tabular}{|c|c|c|c|c|c|c|c|c|c|c|c|c|}
\hline \multirow{2}{*}{$\begin{array}{c}\text { Group } \\
\text { Mouse No. }\end{array}$} & \multicolumn{6}{|c|}{ Control $\left[2^{\text {nd }} L\right]$ group } & \multicolumn{6}{|c|}{ CPA group } \\
\hline & 1 & 2 & 3 & 4 & 5 & 6 & 1 & 2 & 3 & 4 & 5 & 6 \\
\hline 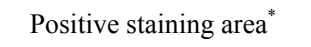 & + & +++ & ++ & ++ & ++ & ++ & +++ & +++ & +++ & +++ & +++ & +++ \\
\hline Positive staining strength ${ }^{*}$ & ++ & ++ & ++ & ++ & ++ & + & ++ & +++ & +++ & ++ & +++ & +++ \\
\hline
\end{tabular}

IHC was evaluated by scoring the positive staining area and positive staining strength of each mouse in the CPA and control [ $\left.2^{\text {nd }} \mathrm{L}\right]$ groups. Scores are represented as: + , slightly positive; ++ , moderately positive; +++ , markedly positive. The statistical analysis was performed after quantification of the scores as described in Materials and Methods. ${ }^{*}$ p $<0.05$ CPA vs control group by Wilcoxon test.
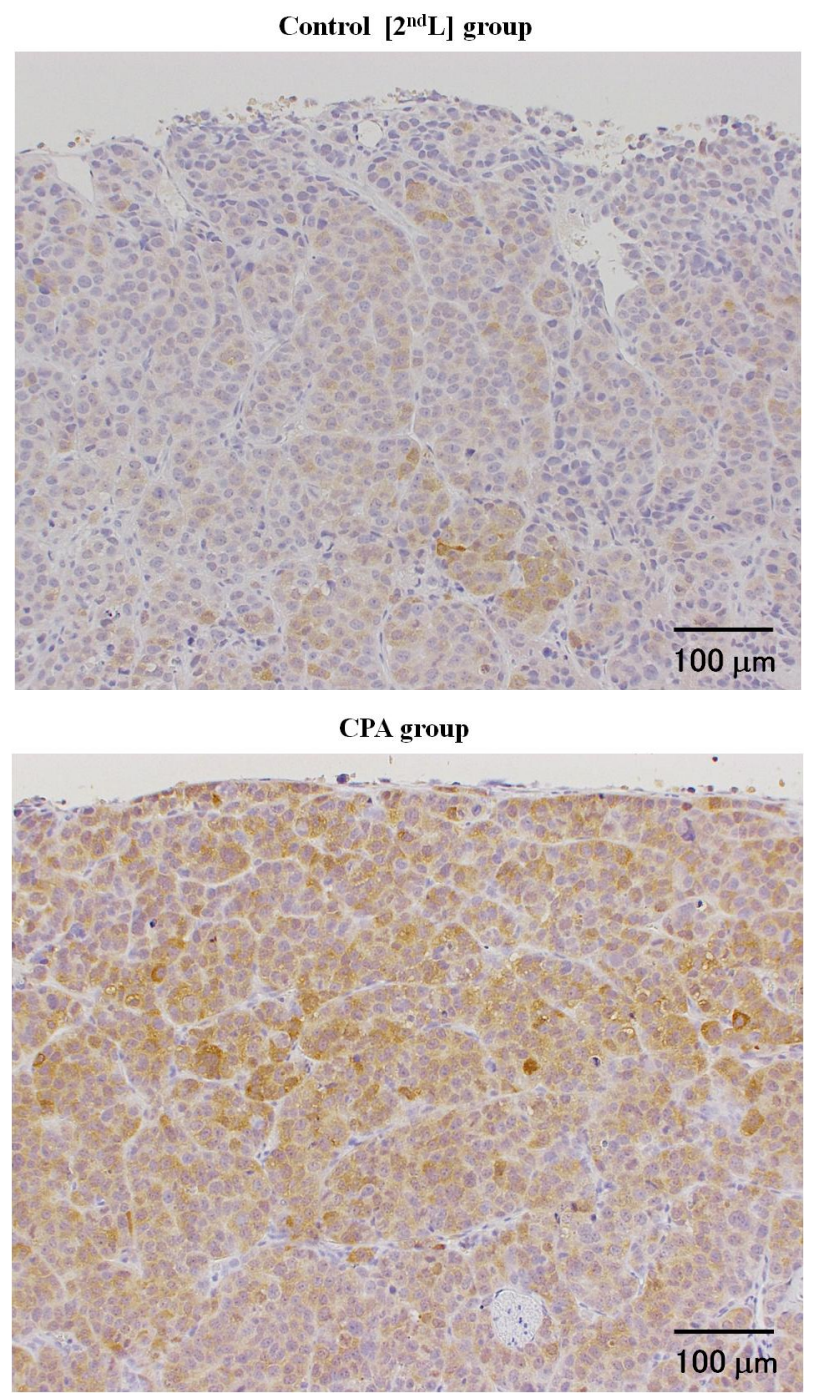

Figure 2. IHC of TP in tumor tissues from mice treated with CPA or vehicle as the $2^{\text {nd }}$-line treatment. Mice were treated as described in Figure 1. The selected mice were randomized into control $\left[2{ }^{\text {nd }} \mathrm{L}\right]$ and CPA groups of 6 mice each on day 36. CPA or DW as a vehicle was given daily for 14 days. The tumors were collected on day 50 for IHC of TP.

mor activity of PTX $+\mathrm{BEV}$ was stronger than that of PTX alone or BEV alone [8]. It has also been reported that CPA upregulated TP in tumors and the CAPE +
CPA combination showed a synergistic antitumor activity as a $1^{\text {st }}$-line treatment in the MX-1 xenograft model [15]. These clinical and preclinical findings prompted us to examine the antitumor efficacy of the CAPE + CPA combination as a $2^{\text {nd }}$-line therapy after PTX $+\mathrm{BEV} 1^{\text {st }}$ line treatment in an MX-1 TNBC xenograft model.

During the $1^{\text {st }}$-line treatment, an obvious antitumor effect was observed in the PTX + BEV group in a similar way as previously reported [8]. However, when the $1^{\text {st }}$ line treatment was prolonged, tumors started to grow. At present, it is unclear why the once-regressed tumors tended to grow even though the treatment was still continued. One explanation may be that the tumors acquired resistance to PTX and/or BEV. The PTX resistance may be caused in part by molecules responsible for multidrug resistance, because it has been reported that the degree of expression in P-glycoprotein/P-gp or multidrug resistance-associated protein $3 / \mathrm{MRP} 3$ affects the resistance to various cancer drugs, including taxanes [21,22]. As for the resistance to antiangiogenic therapy, the following mechanisms have been proposed: upregulation of alternative pro-angiogenic signaling pathways that include fibroblast growth factor, PlGF, ephrin, angiopoietin, or the Notch ligand/receptor system; recruitment of bone marrow-derived cells that secrete numerous angiogenic factors; and increased pericyte coverage of tumor blood vessels to support vasculature [23,24]. However, because there is at present no specifically defined marker for $\mathrm{BEV}$ resistance $[25,26]$, we speculate that the tumor regrowth during the $1^{\text {st }}$-line treatment $(\mathrm{PTX}+\mathrm{BEV})$ in our experiment may be caused by one or more of the above mechanisms.

In the $2^{\text {nd }}$-line treatment, CAPE or CPA as a single agent showed a significant antitumor activity even though the $2^{\text {nd }}$-line treatment had been started when tumors were in the growing phase (day 36). This implies that resistant mechanisms affecting the antitumor efficacy of CAPE or CPA were not induced during $1^{\text {st }}$-line treatment in our model. As explained above, the final step in the conversion of CAPE to 5-FU is governed by $\mathrm{TP}$, which is highly expressed in tumors, and the correlation of CAPE antitumor activity with tumor levels of TP has been shown $[13,14]$. Even though BEV reportedly induced no significant increase in TP levels in 2 human 
colorectal cancer xenograft models [7], PTX has been reported to increase the level of TP in xenografted tumors [16] and, therefore, the TP level in tumor would be increased by PTX + BEV treatment in the $1^{\text {st }}$-line treatment in our study. However, because antitumor activity gradually receded during the $1^{\text {st }}$-line treatment, the amount of TP induced by PTX might also decrease, if the antitumor activity was attenuated by PTX resistance. To clarify the above hypothesis, the change over time in tumor TP levels in $1^{\text {st }}$-line treatment should be examined. In the $2^{\text {nd }}$-line treatment, CAPE + CPA combination showed an extremely high antitumor activity compared to CAPE or CPA monotherapy. Because the TP level in tumor was upregulated by CPA treatment, the superior antitumor effect of CAPE + CPA combination compared to CAPE monotherapy may be attributed to the increased 5-FU level in tumor tissue caused by facilitated conversion from CAPE by TP. These results are similar to that in the $1^{\text {st }}$-line therapy reported previously [15].

Our preclinical results suggest that the CAPE $+\mathrm{CPA}$ combination therapy may be effective as a $2^{\text {nd }}$-line therapy for progressive disease after PTX + BEV $1^{\text {st }}$-line treatment in TNBC patients.

\section{Acknowledgements}

We thank Dr. Kazushige Mori for helpful discussion and comments regarding the manuscript.

\section{REFERENCES}

[1] L. G. Presta, H. Chen, S. J. O’Connor, V. Chisholm, Y. G. Meng, et al., "Humanization of an Anti-Vascular Endothelial Growth Factor Monoclonal Antibody for the Therapy of Solid Tumors and Other Disorders," Cancer Research, Vol. 57, No. 20, 1997, pp. 4593-4599.

[2] K. J. Kim, B. Li, K. Houck, J. Winer and N. Ferrara, "The Vascular Endothelial Growth Factor Proteins: Identification of Biologically Relevant Regions by Neutralizing Monoclonal Antibodies," Growth Factors, Vol. 7, No.1, 1992, pp. 53-64. doi:10.3109/08977199209023937

[3] Y. Wang, D. Fei, M. Vanderlaan and A. Song, "Biological Activity of Bevacizumab, a Humanized Anti-Vegf Antibody in Vitro," Angiogenesis, Vol. 7, No. 4, 2004, pp. 335-345. doi:10.1007/s10456-004-8272-2

[4] K. J. Kim, B. Li, J. Winer, M. Armanini, N. Gillett, et al., "Inhibition of Vascular Endothelial Growth Factor-Induced Angiogenesis Suppresses Tumour Growth in Vivo," Nature, Vol. 362, No. 6423, 1993, pp. 841-844. doi:10.1038/362841a0

[5] H. P. Gerber and N. Ferrara, "Pharmacology and Pharmacodynamics of Bevacizumab as Monotherapy or in Combination with Cytotoxic Therapy in Preclinical Studies," Cancer Research, Vol. 65, No. 3, 2005, pp. 671-680.

[6] P. V. Dickson, J. B. Hamner, T. L. Sims, C. H. Fraga, C.
Y. Ng, et al., "Bevacizumab-Induced Transient Remodeling of the Vasculature in Neuroblastoma Xenografts Results in Improved Delivery and Efficacy of Systemically Administered Chemotherapy," Clinical Cancer Research, Vol. 13, No. 13, 2007, pp. 3942-3950. doi:10.1158/1078-0432.CCR-07-0278

[7] M. Yanagisawa, K. Fujimoto-Ouchi, K. Yorozu, Y. Yamashita and K. Mori, "Antitumor Activity of Bevacizumab in Combination with Capecitabine and Oxaliplatin in Human Colorectal Cancer Xenograft Models," Oncology Reports, Vol. 22, No. 2, 2009, pp. 241-247.

[8] M. Yanagisawa, K. Yorozu, M. Kurasawa, K. Nakano, K. Furugaki, et al., "Bevacizumab Improves the Delivery and Efficacy of Paclitaxel," Anticancer Drugs, Vol. 21, No. 7, 2010, pp. 687-694.

[9] Y. Yamashita-Kashima, K. Fujimoto-Ouchi, K. Yorozu, M. Kurasawa, M. Yanagisawa, et al., "Biomarkers for Antitumor Activity of Bevacizumab in Gastric Cancer Models," BMC Cancer, Vol. 12, 2012, p. 37. doi:10.1186/1471-2407-12-37

[10] S. B. Horwitz, "Mechanism of Action of Taxol," Trends in Pharmacological Sciences, Vol. 13, No. 4, 1992, pp. 134-136. doi:10.1016/0165-6147(92)90048-B

[11] K. Miller, M. Wang, J. Gralow, M. Dickler, M. Cobleigh, et al., "Paclitaxel plus Bevacizumab versus Paclitaxel Alone for Metastatic Breast Cancer," New England Journal of Medicine, Vol. 357, No. 26, 2007, pp. 2666-2676. doi:10.1056/NEJMoa072113

[12] M. Tanaka, Y. Takamatsu, K. Anan, S. Ohno, R. Nishimura, et al., "Oral Combination Chemotherapy with Capecitabine and Cyclophosphamide in Patients with Metastatic Breast Cancer: A Phase II Study," Anti-Cancer Drugs, Vol. 21, No. 4, 2010, pp. 453-458. doi:10.1097/CAD.0b013e328336acb1

[13] H. Yasuno, M. Kurasawa, M. Yanagisawa, Y. Sato, N. Harada, et al., "Predictive Markers of Capecitabine Sensitivity Identified from the Expression Profile of Pyrimidine Nucleoside-Metabolizing Enzymes," Oncology Reports, Vol. 29, No. 2, 2013, pp. 451-458.

[14] T. Ishikawa, F. Sekiguchi, Y. Fukase, N. Sawada and H. Ishitsuka, "Positive Correlation between the Efficacy of Capecitabine and Doxifluridine and the Ratio of Thymidine Phosphorylase to Dihydropyrimidine Dehydrogenase Activities in Tumors in Human Cancer Xenografts," Cancer Research, Vol. 58, No. 4, 1998, pp. 685690.

[15] M. Endo, N. Shinbori, Y. Fukase, N. Sawada, T. Ishikawa, et al., "Induction of Thymidine Phosphorylase Expression and Enhancement of Efficacy of Capecitabine or 5'-Deoxy-5-Fluorouridine by Cyclophosphamide in Mammary Tumor Models," International Journal of Cancer, Vol. 83, No. 1, 1999, pp. 127-134. doi:10.1002/(SICI)1097-0215(19990924)83:1<127::AID$\underline{\mathrm{IJC} 22>3.0 . \mathrm{CO} ; 2-6}$

[16] N. Sawada, T. Ishikawa, Y. Fukase, M. Nishida, T. Yoshikubo, et al., "Induction of Thymidine Phosphorylase Activity and Enhancement of Capecitabine Efficacy by Taxol/Taxotere in Human Cancer Xenografts," Clinical 
Cancer Research, Vol. 4, No. 4, 1998, pp. 1013-1019.

[17] J. Cassidy, J. Tabernero, C. Twelves, R. Brunet, C. Butts, et al., "Xelox (Capecitabine plus Oxaliplatin): Active First-Line Therapy for Patients with Metastatic Colorectal Cancer," Journal of Clinical Oncology, Vol. 22, No. 11, 2004, pp. 2084-2091. doi:10.1200/JCO.2004.11.069

[18] N. Sawada, K. Kondoh and K. Mori, "Enhancement of Capecitabine Efficacy by Oxaliplatin in Human Colorectal and Gastric Cancer Xenografts," Oncology Reports, Vol. 18, No. 4, 2007, pp. 775-778.

[19] K. Fujimoto-Ouchi, M. Yanagisawa, F. Sekiguchi and Y. Tanaka, "Antitumor Activity of Erlotinib in Combination with Capecitabine in Human Tumor Xenograft Models," Cancer Chemotherapy and Pharmacology, Vol. 57, No. 5, 2006, pp. 693-702. doi:10.1007/s00280-005-0079-3

[20] N. Sawada, T. Ishikawa, F. Sekiguchi, Y. Tanaka and H. Ishitsuka, "X-Ray Irradiation Induces Thymidine Phosphorylase and Enhances the Efficacy of Capecitabine (Xeloda) in Human Cancer Xenografts," Clinical Cancer Research, Vol. 5, 1999, pp. 2948-2953.

[21] E. Mechetner, A. Kyshtoobayeva, S. Zonis, H. Kim, R. Stroup, et al., "Levels of Multidrug Resistance (mdr1) p-Glycoprotein Expression by Human Breast Cancer Cor- relate with in Vitro Resistance to Taxol and Doxorubicin," Clinical Cancer Research, Vol. 4, 1998, pp. 389 398.

[22] J. P. de Hoon, J. Veeck, B. E. Vriens, T. G. Calon, M. van Engeland, et al., "Taxane Resistance in Breast Cancer: A Closed Her2 Circuit?" Biochimica et Biophysica Acta, Vol. 1825, No. 2, 2012, pp. 197-206.

[23] A. K. Koutras, I. Starakis, U. Kyriakopoulou, P. Katsaounis, A. Nikolakopoulos, et al., "Targeted Therapy in Colorectal Cancer: Current Status and Future Challenges," Current Medicinal Chemistry, Vol. 18, No. 11, 2011, pp. 1599-1612. doi:10.2174/092986711795471338

[24] S. Tejpar, H. Prenen and M. Mazzone, "Overcoming Resistance to Antiangiogenic Therapies," Oncologist, Vol. 17, No. 8, 2012, pp. 1039-1050. doi:10.1634/theoncologist.2012-0068

[25] N. Ferrara and R. S. Kerbel, "Angiogenesis as a Therapeutic Target," Nature, Vol. 438, No. 7070, 2005, pp. 967-974. doi:10.1038/nature04483

[26] M. F. Mulcahy, "Bevacizumab in the Therapy for Refractory Metastatic Colorectal Cancer," Biologics, Vol. 2, No. 1, 2008, pp. 53-59. 